\title{
Short and zero baseline analysis of GPS L1 C/A, L5Q, GIOVE E1B, and E5aQ signals
}

\author{
Peter F. de Bakker · Christian C. J. M. Tiberius • \\ Hans van der Marel $\cdot$ Roel J. P. van Bree
}

Received: 25 March 2010/Accepted: 5 January 2011/Published online: 5 February 2011

(c) The Author(s) 2011. This article is published with open access at Springerlink.com

\begin{abstract}
Stochastic properties of GNSS range measurements can accurately be estimated using a geometry-free short and zero baseline analysis method. This method is now applied to dual-frequency measurements from a new field campaign. Results are presented for the new GPS L5Q and GIOVE E5aQ wideband signals, in addition to the GPS L1 C/A and GIOVE E1B signals. As expected, the results clearly show the high precision of the new signals, but they also show, rather unexpectedly, significant, slowly changing variations in the pseudorange code measurements that are probably a result of strong multipath interference on the data. Carrier phase measurement noise is assessed on both frequencies, and finally successful mixed GPS-GIOVE double difference ambiguity resolution is demonstrated.
\end{abstract}

Keywords GPS · GIOVE - Multi-constellation ·

Geometry-free $\cdot$ Short baseline $\cdot$ Zero baseline $\cdot$ Multipath . Code noise $\cdot$ Phase noise - Undifferenced $\cdot$ Single, double, triple difference

$\begin{array}{ll}\text { Abbreviations } \\ \text { UD } & \text { Undifferenced } \\ \text { SD } & \text { Single difference } \\ \text { DD } & \text { Double difference } \\ \Delta & \text { Time difference } \\ \text { SB } & \text { Short baseline } \\ \text { ZB } & \text { Zero baseline } \\ \text { Rx } & \text { Receiver }\end{array}$

P. F. de Bakker $(\bowtie)$ - C. C. J. M. Tiberius · H. van der Marel ·

R. J. P. van Bree

Delft University of Technology, Delft,

The Netherlands

e-mail: p.f.debakker@tudelft.nl

\section{Introduction}

A versatile short and zero baseline analysis method was introduced in De Bakker et al. (2009) which uses a geometryfree model and different linear combinations of GNSS measurements to separate the contributions of different error sources to the measurement error at the receiver. The method was applied to measurements collected with two single-frequency Septentrio AsteRx1 receivers tracking satellites from GPS, EGNOS, and GIOVE on the L1/E1 frequency. The method is now applied to dual-frequency data from a new measurement campaign with GPS and includes both GIOVE-A and GIOVE-B. We analyze and address in particular the measurements on the wideband signals at the L5/E5a frequency. In addition, we consider the well-known multipath linear combination, which relies on dual-frequency carrier phase data. We not only study random errors in the data, but also systematic errors which dominate the data behavior over longer timescales. Finally, the geometry-free ambiguity resolution is attempted with the new wideband signals, and the impact of systematic errors on the ambiguity resolution success rate is assessed.

\section{Measurement campaign}

The measurement campaign consists of two measurement setups. A zero baseline was measured on May 29, 2009 with two Septentrio PolaRx3G receivers connected to a single Leica AR25 3D choke ring antenna on top of a 14-floor building. A short baseline was measured on June 1, 2009, with the same two Septentrio PolaRx3G receivers each connected to its own Leica AR25 3D choke ring antenna installed on tripods in the open field about 6 meters separated. 
GIOVE E1B and E5aQ signals were tracked from GIOVE-A and B, and the GPS L1 C/A and L5Q signals were tracked from GPS SVN49 (PRN01). Additionally, the GPS L1 C/A signal was tracked from all other visible GPS satellites. Measurements were collected at a 1-s interval and stored in RINEX-format version 3 (Gurtner and Estey 2007). For further details on the measurement campaign, the reader is referred to Tiberius et al. (2009).

In order to compare and to provide an independent check of our findings, we also processed UNAVCO measurements, contributors to the International GNSS Service (Dow et al. 2009), of the L1 C/A, L2C, and L5Q signals transmitted on June 1, 2009 by GPS SVN49. These GPSonly data were collected with a 76-channel Trimble NetR8 receiver able to track modernized GPS L2C and L5 signals (Trimble Navigation Limited 2008a), and a Trimble GNSS choke ring antenna that supports all GPS frequency bands (Trimble Navigation Limited 2008b). The receiver was located on the roof of the UNAVCO headquarters in Boulder, Colorado, USA.

\section{Methodology}

Our analysis method uses linear combinations of the pseudorange code and carrier phase measurements to characterize the stochastic properties of these measurements. The method has been introduced in De Bakker et al. (2009), Van der Marel et al. (2009), and Tiberius et al. (2009). Table 1 summarizes the single-frequency linear combinations for the code and carrier measurements. The linear combinations (1.c.) considered are the Code-minusCarrier (CC) combination in undifferenced (UD), betweenreceiver single differenced (SD), double differenced (DD), and time-differenced $(\Delta)$ form, and now also the multipath combination (MP) which can be constructed from multifrequency data. It is introduced below in undifferenced and time-differenced form. Table 1 also shows the expectation and dispersion values of each combination for three different measurements setups: single receiver $(1 \mathrm{Rx})$, short baseline (SB), and zero baseline (ZB).

Column five (expectation) shows the systematic effects that are present in each of the linear combinations. These include ionospheric delay $I$, hardware delays $\xi$, code multipath $m p$, and carrier phase ambiguity $A$ if real-valued or $\lambda N$ if integer-valued, where $N$ is the integer number and $\lambda$ the wavelength. Even though multipath is a very complex phenomenon that is difficult to predict, it is considered a systematic effect in our approach. The undifferenced CC combination contains the ionospheric delay; a second order polynomial is fitted typically over a $120 \mathrm{~s}$ time span and subtracted to remove this effect. The ambiguity terms which are present in some of the linear combinations are removed by subtracting the mean value, as indicated in column six (correction) of Table 1, using again a 120 s time span.

Column seven (dispersion) contains the random effects that are present in each of the linear combinations. This is the random measurement noise with variance $\sigma_{C}^{2}$ for the pseudorange code impacted by correlation of the measurements. Two important types of correlation are considered for the code measurements. These are time correlation $\rho_{\Delta}$, and correlation between the code measurements of the two receivers in the zero baseline setup $\rho_{\mathrm{SD}}$ (De Bakker et al. 2009). Accurate estimates of the variance and the correlation coefficients are of great interest for positioning and integrity monitoring, since they can be used to define the stochastic model needed for optimal results. To determine these parameters, the linear combinations in Table 1 are divided into four groups based on their dispersion and remaining systematic effects.

Table 1 Expectation and dispersion values of different linear combinations

\begin{tabular}{|c|c|c|c|c|c|c|}
\hline Group & Setup & Difference & 1.c. & Expectation & Correction & Dispersion \\
\hline \multirow[t]{4}{*}{1} & $1 \mathrm{Rx}$ & UD & $\mathrm{CC}$ & $2 I-A+m p+\xi$ & Polynomial & $\sigma_{C}^{2}$ \\
\hline & $1 \mathrm{Rx}$ & UD & MP & $m p+A_{M P}+\xi_{M P}$ & Mean & $\sigma_{C}^{2}$ \\
\hline & SB & $\mathrm{SD}$ & $\mathrm{CC}$ & $m p_{S D}-A_{S D}+\xi_{S D}$ & Mean & $2 \sigma_{C}^{2}$ \\
\hline & $\mathrm{SB}$ & $\mathrm{DD}$ & $\mathrm{CC}$ & $-\lambda N_{D D}+m p_{D D}$ & Mean & $4 \sigma_{C}^{2}$ \\
\hline \multirow[t]{4}{*}{2} & $1 \mathrm{Rx}$ & $\Delta$ & $\mathrm{CC}$ & $2 \Delta I+\Delta m p$ & - & $2\left(1-\rho_{\Delta}\right) \sigma_{C}^{2}$ \\
\hline & $1 \mathrm{Rx}$ & $\Delta$ & MP & $\Delta m p$ & - & $2\left(1-\rho_{\Delta}\right) \sigma_{C}^{2}$ \\
\hline & $\mathrm{SB}$ & $\Delta \mathrm{SD}$ & $\mathrm{CC}$ & $\Delta m p_{S D}$ & - & $4\left(1-\rho_{\Delta}\right) \sigma_{C}^{2}$ \\
\hline & SB & $\Delta \mathrm{DD}$ & $\mathrm{CC}$ & $\Delta m p_{D D}$ & - & $8\left(1-\rho_{\Delta}\right) \sigma_{C}^{2}$ \\
\hline \multirow[t]{2}{*}{3} & $\mathrm{ZB}$ & $\mathrm{SD}$ & $\mathrm{CC}$ & $-A_{S D}+\xi_{S D}$ & Mean & $2\left(1-\rho_{S D}\right) \sigma_{C}^{2}$ \\
\hline & $\mathrm{ZB}$ & $\mathrm{DD}$ & $\mathrm{CC}$ & $-\lambda N_{D D}$ & Mean & $4\left(1-\rho_{S D}\right) \sigma_{C}^{2}$ \\
\hline \multirow[t]{2}{*}{4} & $\mathrm{ZB}$ & $\Delta \mathrm{SD}$ & $\mathrm{CC}$ & 0 & - & $4\left(1-\rho_{\Delta}\right)\left(1-\rho_{S D}\right) \sigma_{C}^{2}$ \\
\hline & $\mathrm{ZB}$ & $\Delta \mathrm{DD}$ & $\mathrm{CC}$ & 0 & - & $8\left(1-\rho_{\Delta}\right)\left(1-\rho_{S D}\right) \sigma_{C}^{2}$ \\
\hline
\end{tabular}


It may seem straightforward to determine the variance of the random thermal measurement noise $\sigma_{C}^{2}$ directly from one of the linear combinations in group 1 of Table 1. However, this is not trivial because systematic multipath effects cannot easily be removed from the measurements. Therefore, an indirect method is used to determine $\sigma_{C}^{2}$ and both correlation parameters from groups 2, 3, and 4 .

Multipath is strongly correlated over time spans of seconds, and therefore it is greatly reduced in time-differenced measurements such as those in group 2. Remaining ionospheric delay and hardware delays can be neglected. Consequently, the variance of the random errors in group 2 can be estimated accurately from the measurements. For zero baselines in groups 3 and 4, the multipath is eliminated by single differencing. This means that the variance of the random errors in groups 3 and 4 can also be estimated from the measurements. In fact, the variance of the measurements will closely represent the dispersion given in Table 1. The estimated values will contain thermal noise as well as correlation between the measurements. Since there are only three unknown parameters $\rho_{\Delta}, \rho_{\mathrm{SD}}$, and $\sigma_{\mathrm{C}}$, they can all be determined from the variance estimates of groups 2,3 , and 4. All computations are performed with respect to a reference $\mathrm{C} / \mathrm{N}_{0}$ of $45 \mathrm{~dB}-\mathrm{Hz}$. A method to estimate the standard deviation of each of the linear combinations for $\mathrm{C} / \mathrm{N}_{0}=45 \mathrm{~dB}-\mathrm{Hz}$ from the measurements is described in De Bakker et al. (2009).

Multipath linear combination

Dual-frequency measurements allow us to extend our analysis to the new GPS L5Q and Galileo E5aQ signals and to form the multipath combinations. If we take the geometry-free measurement equations and generalize them for frequency $j$ we have:

$$
\begin{aligned}
& C_{j}=g+\frac{f_{i}^{2}}{f_{j}^{2}} I_{i}+m p_{C, j}+\xi_{C, j}+\varepsilon_{C, j} \\
& L_{j}=g-\frac{f_{i}^{2}}{f_{j}^{2}} I_{i}+m p_{L, j}+A_{j}+\xi_{L, j}+\varepsilon_{L, j}
\end{aligned}
$$

where $C$ and $L$ are the code and phase measurements, $g$ is a geometric term which includes the geometric range between the receiver and satellite, the troposphere delay, the receiver clock error, and the satellite clock error. The symbol $f$ denotes the carrier frequency, $I_{i}$ is the ionosphere delay on reference frequency $i, m p_{\mathrm{C}}$ and $m p_{\mathrm{L}}$ are the code and phase multipath, $A$ is the phase ambiguity, $\xi_{\mathrm{C}}$ and $\xi_{\mathrm{L}}$ are the instrumental code and phase delays, and $\varepsilon_{\mathrm{C}}$ and $\varepsilon_{\mathrm{L}}$ are random code and phase measurement errors, respectively. All quantities are expressed in meters except frequency $f$ is in Hertz.

Using dual-frequency phase measurements and a singlefrequency code measurement of one receiver, we can eliminate the first-order ionospheric effect as well as the geometric term $g$ from (1) with the multipath combination:

$M P_{j i}=C_{j}-\frac{f_{j}^{2}+f_{i}^{2}}{f_{j}^{2}-f_{i}^{2}} L_{j}+\frac{2 f_{i}^{2}}{f_{j}^{2}-f_{i}^{2}} L_{i}$

where $i \neq j$. If the phase noise and phase multipath are neglected, this leads to the following expectation and dispersion values of the multipath combination:

$E\left\{M P_{j i}\right\} \approx m p_{C, j}+\xi_{C, j}-\frac{f_{j}^{2}+f_{i}^{2}}{f_{j}^{2}-f_{i}^{2}}\left(A_{j}+\xi_{L, j}\right)+\frac{2 f_{i}^{2}}{f_{j}^{2}-f_{i}^{2}}\left(A_{i}+\xi_{L, i}\right)$

$D\left\{M P_{j i}\right\} \approx \sigma_{C j}^{2}$

The MP combination (3) contains code multipath, a constant ambiguity term which is a combination of the ambiguities of the two phase measurements, a combined hardware delay and thermal noise. Subtraction of the mean value from the measurements removes the phase ambiguities, which are constant if there are no cycle slips. The behavior of receiver hardware delays was studied by Liu et al. (2004), with reported values for the hardware delay change rates under normal conditions below $0.1 \mathrm{~mm} / \mathrm{s}$. Satellite hardware delay change rates are assumed to be constrained to even smaller values. Therefore, the remaining differential hardware delays in (3) will have little impact on short timescales. The resulting time series is dominated by code multipath and thermal noise. With the measured dual-frequency data, two MP combinations can be formed: one for the code on L1/E1 and one for the code on L5/E5a. For the triple-frequency UNAVCO data, many more MP-combinations can be formed with different, some very large, multiplication factors. We have used $\mathrm{MP}_{15}, \mathrm{MP}_{21}$, and $\mathrm{MP}_{51}$ which have relatively small multiplication factors and therefore are less impacted by phase errors.

When differencing consecutive epochs, the constant ambiguity terms are eliminated and the slowly changing hardware delays are greatly reduced. This leads to the following expectation and dispersion for the time differences:

$$
\begin{aligned}
& E\left\{\Delta M P_{j i}\right\} \approx \Delta m p_{C, j} \\
& D\left\{\Delta M P_{j i}\right\} \approx 2\left(1-\rho_{\Delta}\right) \sigma_{C j}^{2}
\end{aligned}
$$

For the measured 1-Hz data, the time-differenced multipath is very small and the residual time series mainly shows the random effects captured in the dispersion. This dispersion depends on the thermal noise and the time correlation.

\section{Results}

First the random measurement noise on the new wideband signals is analyzed, then the remaining systematic 
measurement errors in the linear combinations are highlighted, and finally, ambiguity resolution with the geometry-free model is treated.

\section{Random measurement noise}

Table 2 shows the standard deviations of the measurement residuals on the signals tracked during the short baseline measurements in the field and the zero baseline measurements on the roof for each of the discussed linear combinations. The measured time series were split into data segments of $120 \mathrm{~s}$, and the standard deviation for each of these segments was determined. Based on these standard deviations, which were measured at different $\mathrm{C} / \mathrm{N}_{0}$, the standard deviation for a $\mathrm{C} / \mathrm{N}_{0}$ of $45 \mathrm{~dB}-\mathrm{Hz}$ was estimated from all data. The well-known inversely proportional relation between the $\mathrm{C} / \mathrm{N}_{0}$, expressed in ratio- $\mathrm{Hz}$, and the variance of the noise reduces this estimation to a linear regression on a logarithmic scale with only one unknown parameter (De Bakker et al. 2009). Finally, for easy comparison and for this table only, the increase in variance due to differencing has been compensated, assuming zero correlation.

As expected, Table 2 displays a high precision of the GPS L5Q and Galileo E5aQ signals compared to the signals on the L1/E1 frequency. For the GPS L5Q and Galileo E1B and E5aQ signals, the standard deviation of the CC combination is equal to the standard deviation of the MP combination, showing that the ionospheric delay has been

Table 2 Standard deviations of different measurement combinations and signals expressed in meters. The standard deviations are estimated for $\mathrm{C} / \mathrm{N}_{0}=45 \mathrm{~dB}-\mathrm{Hz}$ based on data segments of $120 \mathrm{~s}$ and are normalized to undifferenced level

\begin{tabular}{|c|c|c|c|c|c|c|c|c|}
\hline \multirow{3}{*}{$\begin{array}{l}\text { Linear } \\
\text { Combination }\end{array}$} & \multicolumn{2}{|c|}{ GPS L1 C/A } & \multicolumn{2}{|c|}{ GPS L5Q } & \multicolumn{2}{|c|}{ Galileo E1B } & \multicolumn{2}{|c|}{ Galileo E5aQ } \\
\hline & SB & ZB & SB & $\mathrm{ZB}$ & SB & ZB & SB & ZB \\
\hline & & & & & & & & Roof \\
\hline UD CC & 0.27 & 0.43 & 0.10 & 0.14 & 0.20 & 0.27 & 0.07 & 0.07 \\
\hline UD MP & 0.38 & 0.49 & 0.10 & 0.14 & 0.20 & 0.27 & 0.07 & 0.07 \\
\hline SD CC & 0.28 & & 0.11 & & 0.21 & & 0.07 & \\
\hline DD CC & 0.28 & & & & 0.18 & & 0.07 & \\
\hline$\Delta \mathrm{CC}$ & 0.15 & 0.18 & 0.05 & 0.05 & 0.11 & 0.12 & 0.04 & 0.04 \\
\hline$\Delta \mathrm{MP}$ & 0.20 & 0.20 & 0.05 & 0.05 & 0.11 & 0.12 & 0.04 & 0.04 \\
\hline$\triangle \mathrm{SD} C \mathrm{C}$ & 0.15 & & 0.05 & & 0.11 & & 0.04 & \\
\hline$\triangle \mathrm{DD} C \mathrm{C}$ & 0.15 & & & & 0.10 & & 0.04 & \\
\hline SD CC & & 0.20 & & 0.05 & & 0.16 & & 0.04 \\
\hline DD CC & & 0.19 & & & & 0.14 & & 0.04 \\
\hline$\triangle \mathrm{SD} C \mathrm{C}$ & & 0.13 & & 0.04 & & 0.10 & & 0.03 \\
\hline$\triangle \mathrm{DD} C \mathrm{C}$ & & 0.12 & & & & 0.09 & & 0.03 \\
\hline
\end{tabular}

removed successfully from the $\mathrm{CC}$ by fitting a second-order polynomial without influencing the noise characterization.

For the GPS L1 C/A signal, the results for the CC combination represent the mean value of all tracked GPS satellites, while the MP combination is only formed for SVN49. This explains that we have different results for these combinations in Table $2: 0.38$ vs. 0.27 , and 0.49 vs. 0.43 . For GPS SVN49, the code and carrier phase on L5 could only be measured continuously for relatively high satellite elevation due to the sharp decrease in $\mathrm{C} / \mathrm{N}_{0}$ with elevation for the L5 signal. As a result, the multipath combination for the L1 C/A signal transmitted by SVN49 could also only be determined for high satellite elevation and thus for relatively high $\mathrm{C} / \mathrm{N}_{0}$ values of about $50 \mathrm{~dB}-\mathrm{Hz}$ for this signal. Note, the sharp decrease in $\mathrm{C} / \mathrm{N}_{0}$ with elevation with the demo payload on SVN49 (Marquis et al. 2009) is only present on the L5 frequency. The standard deviation of the $\mathrm{MP}_{15}$ combination for $\mathrm{C} / \mathrm{N}_{0}=45 \mathrm{~dB}-\mathrm{Hz}$ could therefore only be extrapolated from the measured values at higher $\mathrm{C} / \mathrm{N}_{0}$, resulting in an inaccurate estimate.

Comparison of GPS L5Q and Galileo E5aQ results reveals that for undifferenced observations, the GPS L5Q signal has a larger standard deviation by a factor of 1.5-2. This is explicable because the majority of L5Q measurements were collected with high $\mathrm{C} / \mathrm{N}_{0}$. It should be noted that DD GPS L5Q results are not available because at the time of measurement only one GPS satellite transmitted the L5Q signal.

The results in Table 2 allow the estimation of the standard deviation, the time correlation, and the ZB SD correlation as discussed in the previous section. The ZB SD correlation expresses the relation between two different noise contributions, namely amplifier noise, which includes sky and ground noise and which is equal for both receivers in the zero baseline setup, and internal receiver noise (Tiberius et al. 2009). The results from these noise and correlation computations are presented in Table 3. In order to assess the sensitivity of our approach to the length of the data segments, the entire processing has been repeated for data segments of $1,800 \mathrm{~s}$. For this data segment length, the UD CC and MP results are significantly larger than those presented in Table 2, especially for the L5Q and E5aQ

Table 3 Time correlation, zero baseline correlation, measured and theoretical thermal noise on code measurements

\begin{tabular}{lllllll}
\hline & \multicolumn{2}{l}{ GPS } & & \multicolumn{2}{l}{ Galileo } \\
\cline { 2 - 3 } & L1 C/A & L5Q & & E1B & E5aQ \\
\hline$\rho_{\Delta}[-]$ & 0.59 & 0.35 & & 0.60 & 0.43 \\
$\rho_{Z B-S D}[-]$ & 0.51 & 0.28 & & 0.30 & 0.43 \\
Measured $\sigma_{C}[\mathrm{~m}](120 \mathrm{~s}$ segments $)$ & 0.23 & 0.06 & & 0.17 & 0.05 \\
Measured $\sigma_{C}[\mathrm{~m}](1,800$ s segments $)$ & 0.24 & 0.07 & & 0.18 & 0.06 \\
Theoretical $\sigma_{C}[\mathrm{~m}]$ & 0.18 & 0.06 & & 0.11 & 0.06 \\
\hline
\end{tabular}


Fig. 1 Double difference phase measurements of GIOVE-A and $\mathrm{B}$ for E1 and E5a for the short baseline. E5a is tracked here with lower signal power and consequently has more measurement noise
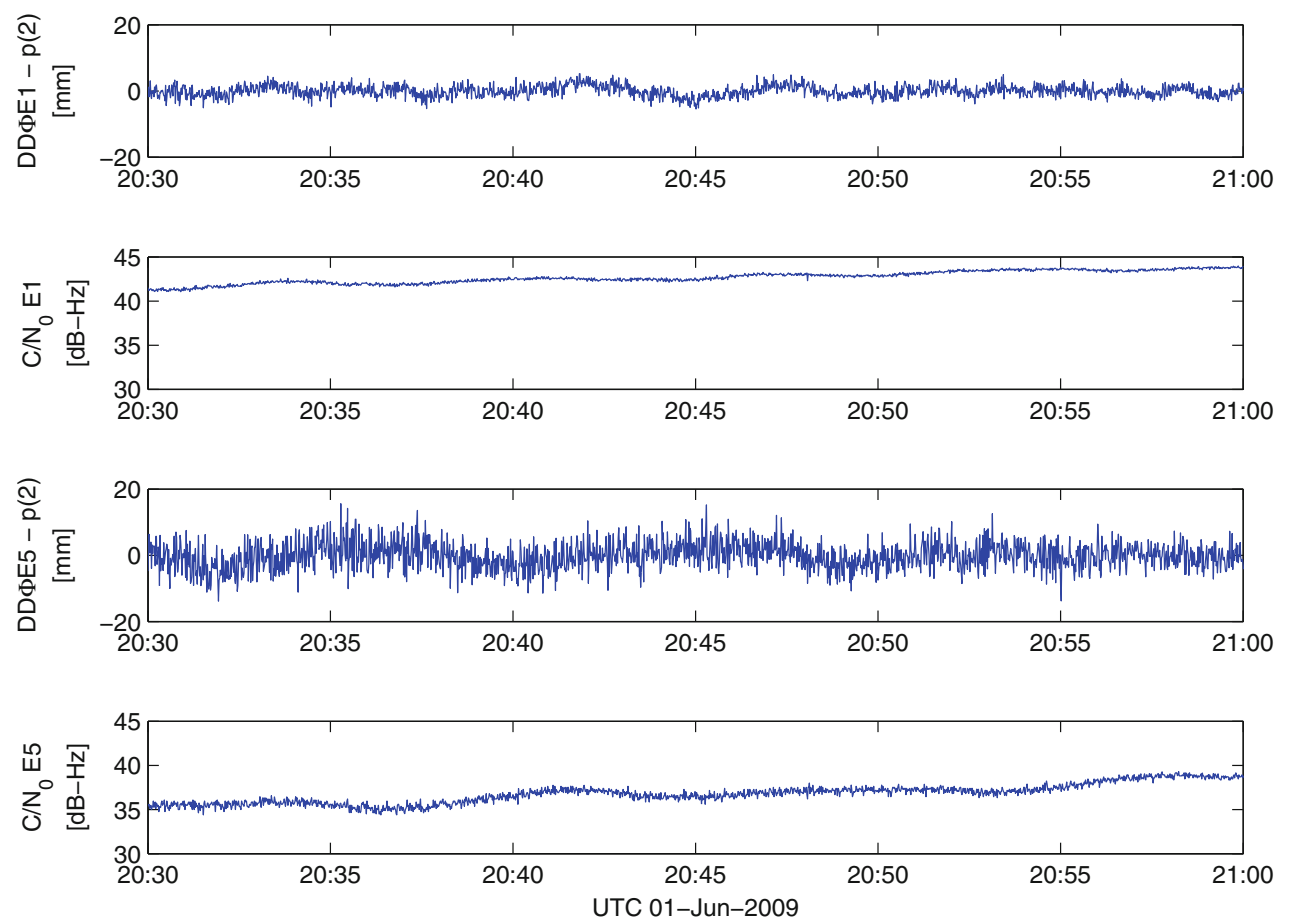

signals. However, such an increase is no longer present in the time-differenced and ZB SD measurements due to the absence of multipath in these combinations. The final estimate of the pseudorange measurement noise is only slightly larger for the longer data segments as shown in Table 3. The correlation values vary little.

Table 3 also shows the expected standard deviation using the theoretical expressions by Braasch and Van Dierendonck (1999) and Sleewaegen et al. (2004), and the receiver and signal properties from Tiberius et al. (2009).

Both the GPS L5Q and the Galileo E5aQ wide band signals have thermal measurement noise on the order of about $6 \mathrm{~cm}$, which is much lower than seen for the E1B and especially the L1 C/A signal.

The carrier phase measurement noise was also analyzed by forming the double difference carrier phase (DDФ) combination. Figure 1 shows part of the DD $\Phi$ time series in panes 1 and 3 and corresponding $C / \mathrm{N}_{0}$ values in panes 2 and 4 for both frequencies of GIOVE-A and B. A secondorder polynomial $p(2)$ has been fitted, between receiver clock jumps to remove the geometric effect and carrier phase ambiguity in the short baseline (SB) setup. The E5a carrier phase measurements are noisier than the E1 carrier phase measurements. This is in small part due to the larger wavelength, but the main reason is the lower received signal power which can be seen in the $\mathrm{C} / \mathrm{N}_{0}$ measurements. An interesting detail is that the $\mathrm{C} / \mathrm{N}_{0}$ measurements themselves are also noisier on E5a than on E1.

Analogously to the pseudorange code noise, the thermal carrier phase noise has been estimated for GPS L1, Galileo
$\mathrm{E} 1$, and Galileo E5a with respect to a $\mathrm{C} / \mathrm{N}_{0}$ of $45 \mathrm{~dB}-\mathrm{Hz}$. Since only one GPS satellite was transmitting the L5Q signal, the GPS L5 carrier phase noise could not be evaluated in this manner. Table 4 shows the results for the DD $\Phi$ and time-differenced DD $\Phi$ for the short and zero baselines. Table 5 shows that the measured values are very

Table 4 Standard deviation of DD and $\triangle \mathrm{DD}$ carrier phase measurements in millimeters for both measurement set-ups, estimated for $\mathrm{C} / \mathrm{N}_{0}=45 \mathrm{~dB}-\mathrm{Hz}$ based on data segments of $120 \mathrm{~s}$ and normalized to undifferenced level

\begin{tabular}{llll}
\hline Linear combination & $\begin{array}{l}\text { GPS } \\
\text { L1 C/A }\end{array}$ & Galileo & \\
\cline { 3 - 4 } & & E1B & E5aQ \\
\hline SB DD $\Phi$ & 0.58 & 0.53 & 0.68 \\
SB $\Delta$ DD $\Phi$ & 0.52 & 0.53 & 0.69 \\
ZB DD $\Phi$ & 0.34 & 0.39 & 0.47 \\
ZB $\Delta$ DD $\Phi$ & 0.34 & 0.40 & 0.48 \\
\hline
\end{tabular}

Table 5 Time correlation, zero baseline correlation, measured and theoretical thermal noise on carrier phase measurements

\begin{tabular}{lcrr}
\hline & GPS & \multicolumn{2}{c}{ Galileo } \\
\cline { 3 - 4 } & L1 C/A & E1B & E5aQ \\
\hline$\rho_{\Delta, \Phi}[-]$ & -0.03 & -0.02 & -0.05 \\
$\rho_{Z B-S D, \Phi}[-]$ & 0.57 & 0.44 & 0.53 \\
Measured $\sigma_{\Phi}[\mathrm{mm}]$ & 0.51 & 0.53 & 0.68 \\
Theoretical $\sigma_{\Phi}[\mathrm{mm}]$ & 0.54 & 0.54 & 0.72 \\
\hline
\end{tabular}


close to the theoretical expectations. There is little time correlation on these $1-\mathrm{Hz}$ phase measurements, but the ZB SD correlation is significant.

\section{Systematic measurement errors}

The expectations in Table 1 show that the residuals of the linear combinations still contain some systematic effects. Most of these effects can either be removed by detrending the data, e.g. the ionospheric delay can be removed by fitting a second-order polynomial to the undifferenced CC measurements, or be neglected for the purposes of this study, e.g. the slowly changing hardware delays. However, not all systematic effects fall into these categories. The most important remaining systematic effect, i.e. multipath, is not removed from the undifferenced residuals or from the short baseline single differences.

Long-term variations in the time series such as those that could result from multipath from a nearby reflector eventually have little impact on the noise characterization through Tables 2 and 3, based on $120 \mathrm{~s}$ data segments. However, multipath dominates the residual time series on longer timescales as can be seen in Fig. 2. Panels a, b, and c show for the short baseline measurements the $\mathrm{MP}_{51}$ combination, the measured $C / \mathrm{N}_{0}$, and the satellite elevation for GIOVE-A, GIOVE-B, and GPS SVN49. The mean value over the full time span has been subtracted to present the variations more clearly. The MP combination shows strong variations in the order of meters over long periods of time. This type of variation was not encountered on the L1/E1 frequency.

These measurements have been carried out with the manufacturer proprietary multipath mitigation intentionally disabled to show the 'raw' multipath effects. For comparison, Fig. 3 shows the $\mathrm{MP}_{51}$ results for GIOVE-B from the roof measurements (ZB). These results are very similar for both receivers, since multipath effects are largely the same for both receivers for a zero baseline. The MP combination again shows strong variations in the order of one meter. The pattern is typical of multipath with the strongest effects at low satellite elevation.

Figure 4 shows the $\mathrm{MP}_{15}$ results for GPS SVN49 and GIOVE-B from the field measurements. The very large variations found in the L5/E5a $\left(\mathrm{MP}_{51}\right)$ results are not observed with $\mathrm{L} 1 / \mathrm{E} 1\left(\mathrm{MP}_{15}\right)$; note that the vertical axis ranges only from $-2 \mathrm{~m}$ to $+2 \mathrm{~m}$. There is a slowly changing elevation-dependent bias on the GPS SVN49 measurements on L1, which was previously reported by Erker et al. (2009). This effect, caused by a signal reflection inside the satellite (Langley 2009), will not significantly influence our noise assessment due to its slow nature, but does show up on the complete L1 C/A time series.

As mentioned above, the UNAVCO measurements of June 1, 2009, were also processed and are presented for comparison. The top and middle panes of Fig. 5 show the multipath combinations in, respectively, undifferenced and time-differenced form of the L1 C/A ( $\left.\mathrm{MP}_{15}\right)$, L2C $\left(\mathrm{MP}_{21}\right)$, and L5Q $\left(\mathrm{MP}_{51}\right)$ signals for GPS SVN49 for this day. The time series of the different frequencies are offset by $2 \mathrm{~m}$ for visual purposes. The bottom pane shows the $\mathrm{C} / \mathrm{N}_{0}$ of the same signals. The MP combinations of each signal show typical code multipath effects at the start and end of the time series, i.e. at low elevation, with amplitudes of a few meters. Multipath effects on the L5Q signal are in the same order of magnitude as those on the other two signals, which means that the higher signal bandwidth does not reduce these effects. In addition, Fig. 5 not only shows the previously mentioned elevation-dependent bias on L1 C/A $\left(\mathrm{MP}_{15}\right)$, but also shows a clear long-term systematic effect on L5Q $\left(\mathrm{MP}_{51}\right)$. This could be caused by elevation-dependent differential hardware delays, or other systematic effects, which are amplified differently in the multipath combinations. The systematic effects are largely eliminated in the time differences, resulting in a white noise-like signal. Due to the 15 -s measurement interval, the residual time differences are significantly larger than those for the PolaRx3G data which was measured at $1 \mathrm{~Hz}$. The measured $\mathrm{C} / \mathrm{N}_{0}$ values for $\mathrm{L} 5 \mathrm{Q}$ show the strong elevation dependence reported by Erker et al. (2009).

Figure 6 shows the standard deviation of the multipath linear combination as a function of the mean satellite elevation for data segments of $600 \mathrm{~s}$ (40 epochs with 15-s measurement interval) for a 7-day period of the UNAVCO measurements of GPS SVN49. The figure shows that the L1 C/A code has the largest range in standard deviation with high precision at high $\mathrm{C} / \mathrm{N}_{0}$, noting that such large values are not reached for the other signals, and low precision for lower $\mathrm{C} / \mathrm{N}_{0}$. The $\mathrm{L} 2 \mathrm{C}$ signal shows a similar precision for midrange $\mathrm{C} / \mathrm{N}_{0}$, but the precision remains better for lower $\mathrm{C} / \mathrm{N}_{0}$. The precision of the L5Q signal is also comparable to the other signals, but this is reached for much smaller values of the $\mathrm{C} / \mathrm{N}_{0}$, which seems to indicate a better performance of this signal.

Figure 7 shows the same data but now as a function of satellite elevation. It does not show this strong improvement of the L5Q signal with respect to the other signals, although the L1 C/A signal still shows the widest range in precision. The connection between Figs. 6 and 7 is formed by the measured $\mathrm{C} / \mathrm{N}_{0}$ as a function of elevation, which mainly depends on the antennas of the receiver and, especially for L5, the satellite. As can be seen, the standard deviation as a function of elevation is very similar for the three signals despite the large differences in $\mathrm{C} / \mathrm{N}_{0}$. This can be explained as follows: the standard deviation for $600 \mathrm{~s}$ data segments with 15 -s measurement interval is not just a 
Fig. $2 \mathrm{MP}_{51}$ combination, $\mathrm{C} / \mathrm{N}_{0}$, and satellite elevation for field observations of a GIOVE-A, b GIOVE-B, and c GPS SVN49
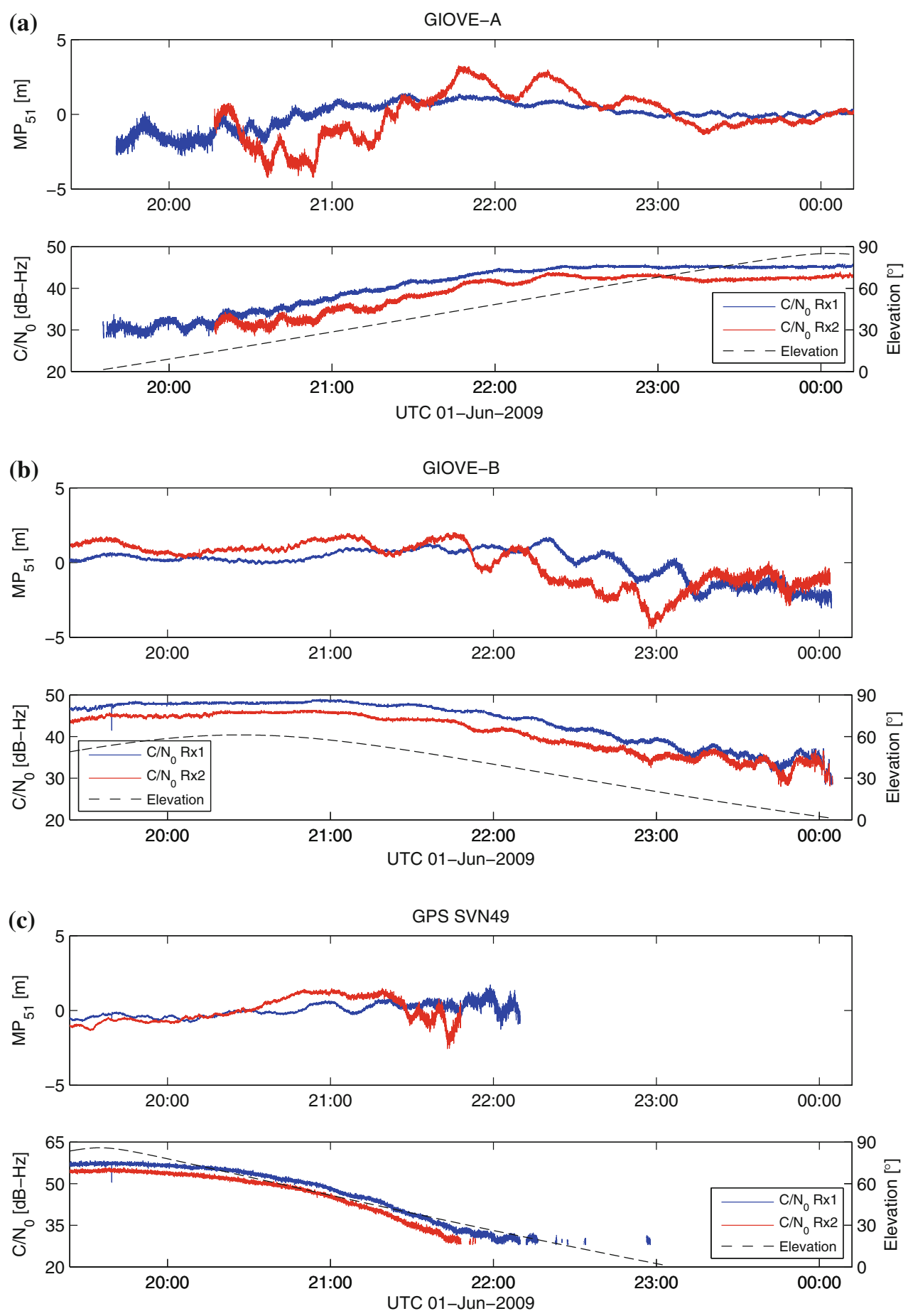

function of the $\mathrm{C} / \mathrm{N}_{0}$, but it is also greatly impacted by multipath. Since multipath strongly depends on elevation, this becomes clearly visible in Fig. 7.

Although these results are greatly affected by the strong elevation dependence of the C/ $\mathrm{N}_{0}$ for L5Q and limited by the 15-s measurement interval, this analysis shows that it remains to be seen whether the expected high performance with the wide band L5Q signal will be realized in practice in the presence of multipath.
The performance of future applications using the wide band signals on E5a and L5 might be severely compromised by the variations on the multipath and code-minuscarrier combinations which were measured in the field. Therefore, a better understanding of their source is desirable. Multipath from a reflector close to the antenna is one effect that could cause long-term variations like those encountered on the CC and MP combinations of the L5/E5a measurements seen in Fig. 2. In Tiberius et al. (2009), this 
Fig. $3 \mathrm{MP}_{51}$ combination, $\mathrm{C} / \mathrm{N}_{0}$, and satellite elevation for GIOVE-B for receiver Rx1 located on the roof

Fig. $4 \mathrm{MP}_{15}$ combination for GPS SVN49 and GIOVE-B for the field measurements

Fig. 5 Multipath combinations, time differences, and $\mathrm{C} / \mathrm{N}_{0}$ values of $\mathrm{L} 1 \mathrm{C} / \mathrm{A}\left(\mathrm{MP}_{15}\right), \mathrm{L} 2 \mathrm{C}$ $\left(\mathrm{MP}_{21}\right)$, and L5Q $\left(\mathrm{MP}_{51}\right)$ from UNAVCO measurements to GPS SVN49 on June 1, 2009. The time series within both the top and the middle panes are offset by 2 meters for visual purposes
GIOVE-B
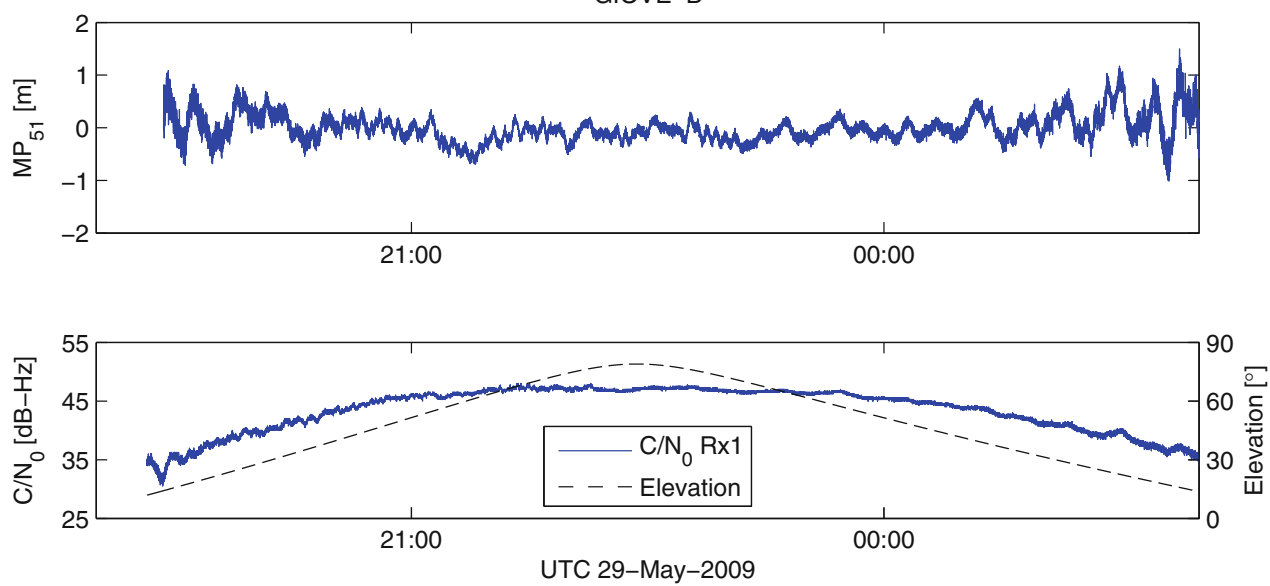

SVN49

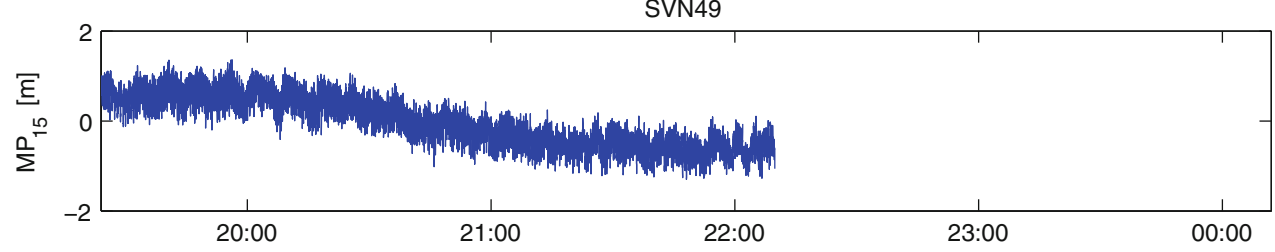

GIOVE-B
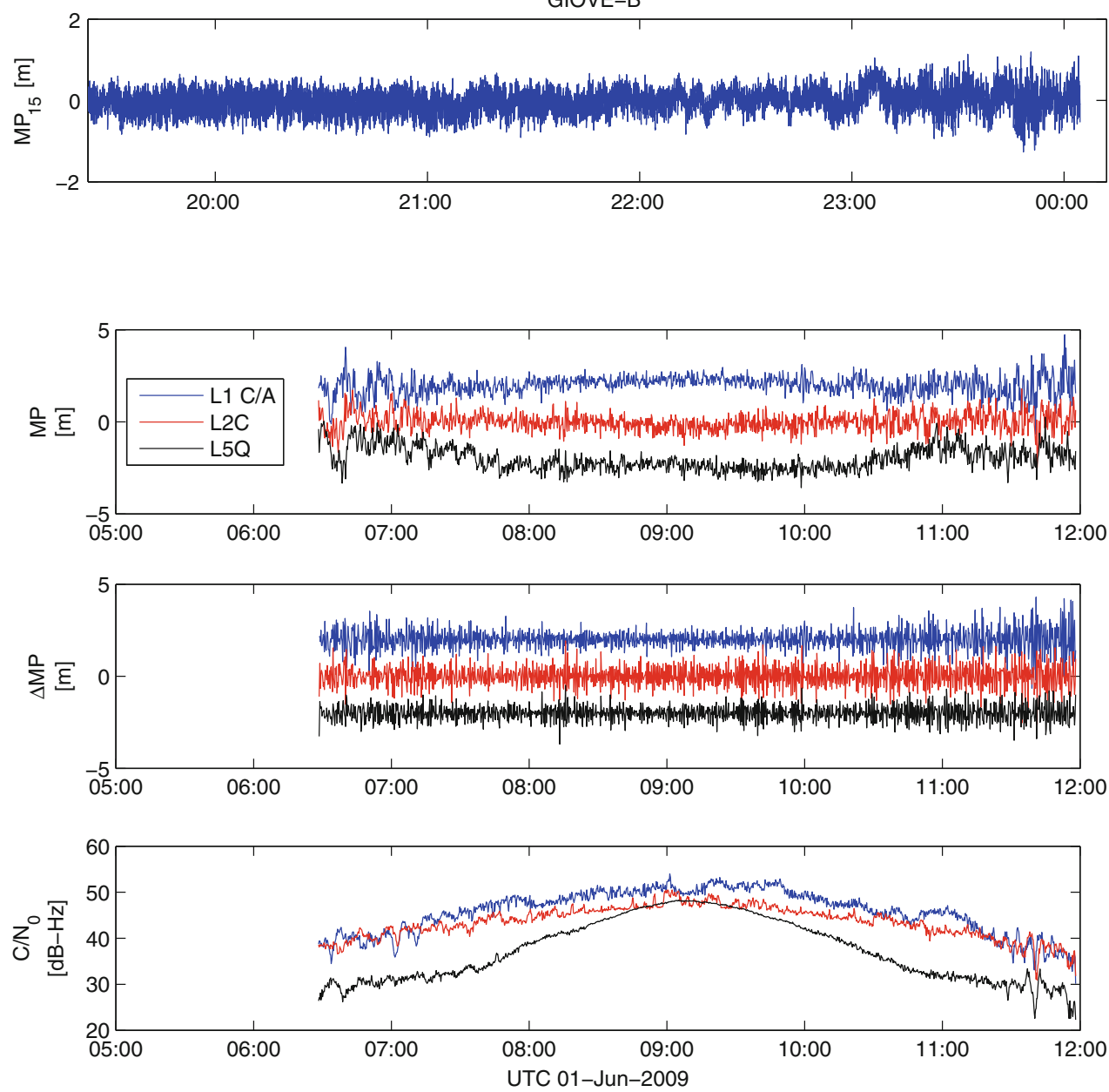


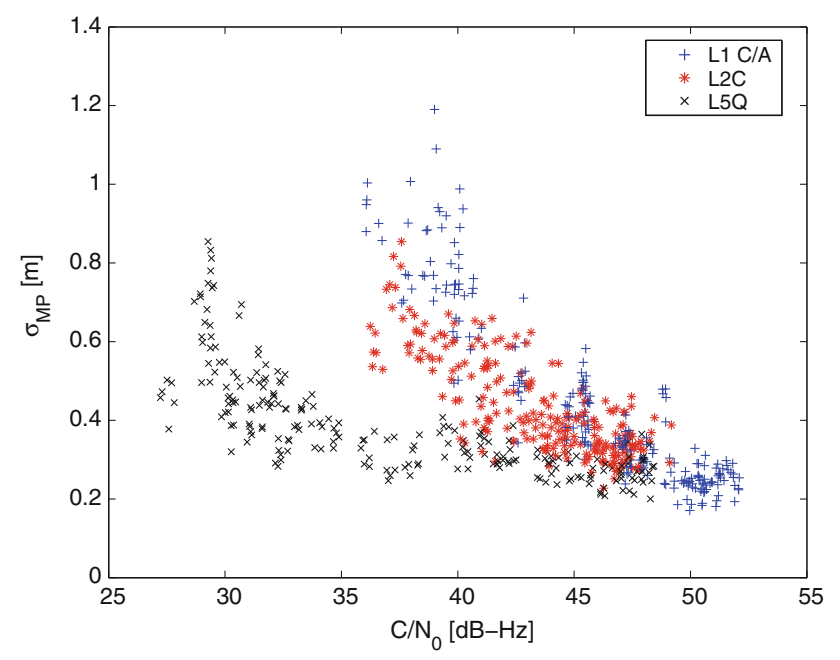

Fig. 6 Multipath combination standard deviation vs. $\mathrm{C} / \mathrm{N}_{0}$ of the $\mathrm{L} 1$ C/A $\left(\mathrm{MP}_{15}\right)$, L2C $\left(\mathrm{MP}_{21}\right)$, and L5Q $\left(\mathrm{MP}_{51}\right)$ signals for GPS SVN49 for June 1-7, 2009, from the UNAVCO measurements. Each marker represents the standard deviation of a data segment of $600 \mathrm{~s}$ (at $1 / 15 \mathrm{~Hz}$ ) at the mean $\mathrm{C} / \mathrm{N}_{0}$ during the segment

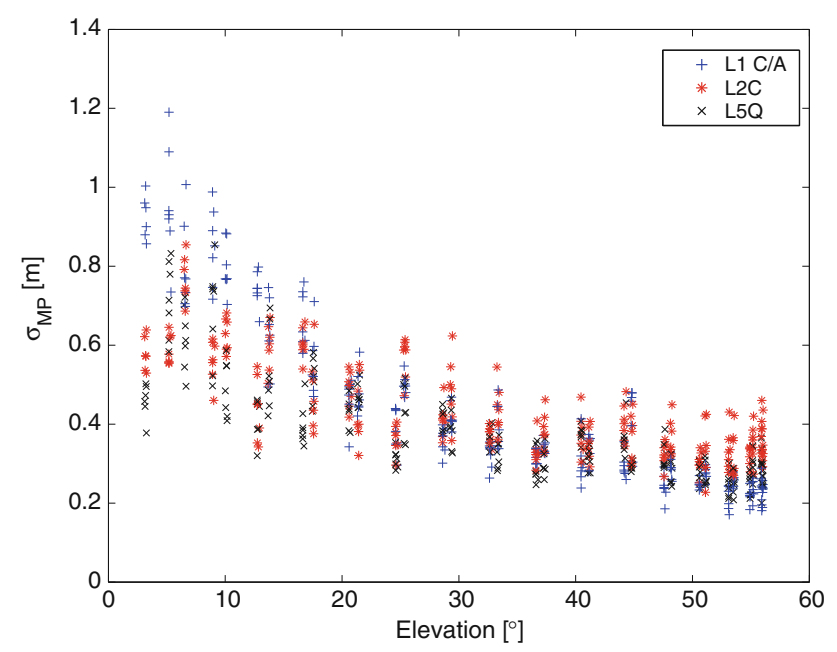

Fig. 7 Multipath combination standard deviation vs. satellite elevation of the L1 C/A $\left(\mathrm{MP}_{15}\right), \mathrm{L} 2 \mathrm{C}\left(\mathrm{MP}_{21}\right)$, and L5Q $\left(\mathrm{MP}_{51}\right)$ signals for GPS SVN49 for June 1-7, 2009, from the UNAVCO measurements. Each marker represents the standard deviation of a data segment of $600 \mathrm{~s}($ at $1 / 15 \mathrm{~Hz})$ at the mean elevation during the segment

was explored in more detail and, although no definite conclusion was reached, the most likely cause is indeed severe multipath in combination with weak multipath rejection of the antenna. Bedford et al. (2009) indicate that in the low-frequency band (L5, E5a, E5b), the Leica AR25 $3 \mathrm{D}$ choke ring antenna has a front-back ratio which is $10 \mathrm{~dB}$ lower than a traditional 2D choke ring antenna. This results from the design trade-off between multipath rejection and low elevation tracking of satellites, the latter of which has significantly improved for the 3D antenna. The front-back ratio indicates the antenna's directivity and resistance to multipath, driven by the antenna's shielding and sensitivity to left-hand circularly polarized signals with respect to the right-hand circularly polarized line-of-sight signals. Slowly changing differential hardware delays can also result in variations in the multipath combinations on very long timescales. Such variations were found in the UNAVCO data (Fig. 5) and might also be present on our own measurements, but there they would not be noticeable due to the very strong variations that are probably caused by multipath.

Geometry-free ambiguity resolution and GNSS inter-operability

Figures 8 and 9 show the SD and DD data for the GIOVE-A and B satellites for the short and zero baselines. The top pane of each figure shows the SD for each of the satellites, while the middle panes show the DD between the satellites. The bottom panes show the DD $\mathrm{C} / \mathrm{N}_{0}$ which is a combination of the $\mathrm{C} / \mathrm{N}_{0}$ for the two satellites and gives a measure for the noise that can be expected in the DD measurements (De Bakker et al. 2009).

From the figures, it is clear that the strong multipath-like variations are not eliminated in the SD or DD for the short baseline, but they are eliminated for the zero baseline (Table 1). This is in line with the expectations for multipath effects.

Table 1 shows that the DD CC combination mainly contains the integer DD ambiguity, code multipath, and code noise. In the mean value of a time series of DD CC, the noise term averages out leaving just the ambiguity and the multipath contributions. In addition, for the zero baseline the multipath is eliminated in the SD and DD differences. This means that the expectation value of the mean of a time series of DD CC measurements, divided by the wavelength, is equal to the integer ambiguity which thus can be determined.

Table 6 shows results of short and zero baseline geometry-free ambiguity resolution on GPS L1, Galileo E1 and E5a, and GPS-Galileo mixed L1/E1 and L5/E5a measurements. For data segments of $30 \mathrm{~s}$ and $600 \mathrm{~s}$, the mean value of DD CC combination minus the true value of the ambiguity was determined and, if the resulting absolute value is smaller than 0.5 , rounding to the nearest integer successfully solves the ambiguity.

Because the measurement noise averages out, the success rate is higher for the longer data segments of $600 \mathrm{~s}$. Due to the elimination of multipath, the success rate is quite high for the zero baseline measurements. For these zero baseline measurements, the ambiguity resolution on the Galileo E5a frequency has an even much higher success rate than on E1 due to better noise characteristics of the wide band signals and the longer E5a carrier wavelength. A similar improvement is visible on the GPS-Galileo 
Fig. 8 Single and Double Difference E5a Code-minusCarrier measurements and DD $\mathrm{C} / \mathrm{N}_{0}$ for GIOVE-A and $\mathrm{B}$ for the short baseline. Successful geometry-free ambiguity resolution is not possible with these measurements

Fig. 9 Single and Double Difference E5a Code-minusCarrier measurements and DD $\mathrm{C} / \mathrm{N}_{0}$ for GIOVE-A and $\mathrm{B}$ on the zero baseline. Successful geometry-free ambiguity resolution is possible with these measurements
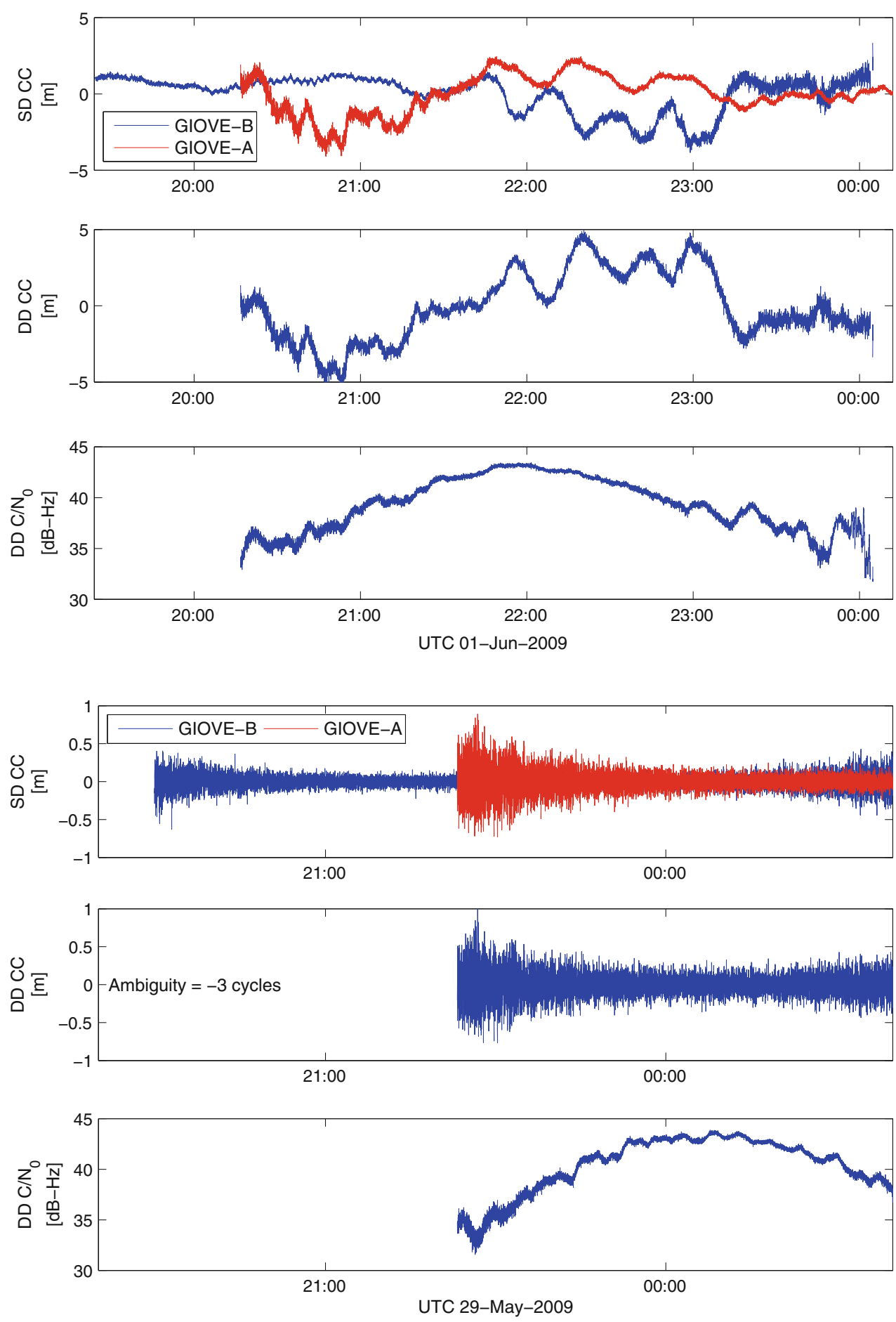

mixed L5/E5a measurements with respect to the L1/E1 performance.

For the short baseline, the DD CC code multipath is not eliminated and multipath in general is not a zero mean process. As a result, the success rate of geometry-free ambiguity resolution on the short baseline is lower. The extreme multipath effects on the L5 frequency completely prevented ambiguity resolution for the short baseline, as the correct ambiguities could not be determined even from the entire time series.

On both the L1/E1 and the L5/E5a frequencies, the mixed GNSS ambiguity resolution performs similarly to the single system ambiguity resolution for both the short baseline and the zero baseline. From this point of view, there seems to be no obstacle for inter-operability between GPS and Galileo in high-precision applications. 
Table 6 Geometry-free ambiguity resolution success rate for GPS, Galileo, and GPS-Galileo mixed ambiguities on L1/E1 and L5/E5a, for the short and zero baselines and for data segments of 30 and $600 \mathrm{~s}$. GPS-only ambiguity resolution on L5 was not possible because there is only one satellite; on the short baseline, ambiguity resolution was not possible due to the strong multipath effects

\begin{tabular}{|c|c|c|c|c|c|c|c|}
\hline & & \multicolumn{2}{|l|}{ GPS } & \multicolumn{2}{|c|}{ Galileo } & \multicolumn{2}{|c|}{ Mixed GPS-Galileo } \\
\hline & & $\begin{array}{l}\mathrm{L} 1 \\
(\%)\end{array}$ & $\begin{array}{l}\text { L5 } \\
(\%)\end{array}$ & $\begin{array}{l}\text { E1 } \\
(\%)\end{array}$ & $\begin{array}{l}\text { E5a } \\
(\%)\end{array}$ & $\begin{array}{l}\text { L1-E1 } \\
(\%)\end{array}$ & $\begin{array}{l}\text { L5-E5a } \\
(\%)\end{array}$ \\
\hline \multirow[t]{2}{*}{ SB } & $30 \mathrm{~s}$ & 27.7 & - & 28.8 & - & 28.0 & - \\
\hline & $600 \mathrm{~s}$ & 61.6 & - & 68.2 & - & 67.9 & - \\
\hline \multirow[t]{2}{*}{ ZB } & $30 \mathrm{~s}$ & 82.4 & - & 76.7 & 99.3 & 77.1 & 100 \\
\hline & $600 \mathrm{~s}$ & 100 & - & 95.7 & 100 & 100 & 100 \\
\hline
\end{tabular}

\section{Concluding remarks}

Short and zero baseline measurements have revealed low thermal noise of about $6 \mathrm{~cm}$ on both the GPS L5Q and the Galileo/GIOVE E5aQ signals which is in line with theoretical expectations for these wide band signals. However, the results also showed strong variations of the pseudorange code measurements over longer time periods, the magnitude of the variations easily reaching up to 20 times the thermal noise standard deviation. Despite being observed in what would generally be considered a friendly multipath environment, the most likely cause of these variations is severe short-range multipath combined with low multipath rejection by the antenna.

Many applications will not be able to take full benefit of the high precision of the new L5Q and E5aQ signals due to the presence of the strong multipath variations encountered on the measurements. The higher precision of the new code observables and the longer wavelength of the L5/E5a carrier with respect to L1/E1 did not lead to the expected increased success rate for geometry-free ambiguity resolution for the short baseline. In fact, ambiguity resolution was not possible for the short baseline measurements. Research into better multipath rejection by L5/E5 capable antennas seems of paramount importance.

The results showed that GPS and Galileo mixed DD ambiguities could be resolved with a success rate comparable to single system ambiguities, which holds great promise for future system inter-operability.

Acknowledgments The authors would like to express their gratitude to UNAVCO for publicly sharing their data collected with Trimble NetR8 receiver tracking the demonstration L5 signal from SVN49.

Open Access This article is distributed under the terms of the Creative Commons Attribution Noncommercial License which permits any noncommercial use, distribution, and reproduction in any medium, provided the original author(s) and source are credited.

\section{References}

Bedford L, Brown N, Walford J (2009) Leica AR25-white paper. Leica Geosystems AG, Heerbrugg, Switzerland, 10 pp

Braasch MS, van Dierendonck AJ (1999) GPS receiver architectures and measurements. Proc IEEE 87(1):48-64

De Bakker PF, van der Marel H, Tiberius CCJM (2009) Geometryfree undifferenced, single and double differenced analysis of single-frequency GPS, EGNOS, and GIOVE-A/B measurements. GPS Solutions, 10 pp. doi: 10.1007/s10291-009-0123-6

Dow JM, Neilan RE, Rizos C (2009) The international GNSS service in a changing landscape of global navigation satellite systems. J Geodesy 83:191-198 (see IGS mail 5967). doi:10.1007/ s00190-008-0300-3

Erker S, Thölert S, Furthner J, Meurer M, Häusler M (2009) GPS L5 "Light's on!"-a first comprehensive signal verification and performance analysis. In: Proceedings of ION GNSS 2009. September 22-25, Savannah, GA, pp 1544-1551

Gurtner W, Estey L (2007) RINEX - the receiver independent exchange format version 3.00. Astronomical Institute, University of Bern - UNAVCO, Boulder

Langley R (2009) The SVN49 pseudorange error. GPS World: 8-14

Liu X, Tiberius C, de Jong K (2004) Modelling of differential single difference receiver clock bias for precise positioning. GPS Solut 7(4):209-221. doi:10.1007/s10291-003-0079-x

Marquis W, McFadden M, Powell T, Irvine J, Erwin B (2009) L5 demo payload-from concept to capability in less than 12 months. GPS World 20(5):27-31

Sleewaegen J-M, de Wilde W, Hollreiser M (2004) Galileo AltBOC receiver. In: Proceedings of ENC-GNSS-2004. Rotterdam, The Netherlands, May, 9 pp

Tiberius CCJM, de Bakker PF, van der Marel H, van Bree RJP (2009) Geometry-free analysis of GIOVE-A/B E1 - E5a, and GPS L1 - L5 measurements. In: Proceedings of ION GNSS 2009, September 22-25, Savannah, GA, pp 2911-2925

Trimble Navigation Limited (2008a) User Guide Trimble NetR8 GNSS Reference Receiver Version 3.80 Revision A. Sunnyvale, CA, USA

Trimble Navigation Limited (2008b) GNSS geodetic antennas brochure. Sunnyvale, CA, USA

Van der Marel H, de Bakker PF, Tiberius CCJM (2009) Zero, single and double difference analysis of GPS, EGNOS and GIOVE-A/B pseudorange and carrier phase measurements. In: Proceedings of ENC GNSS 2009, Naples, Italy

\section{Author Biographies}

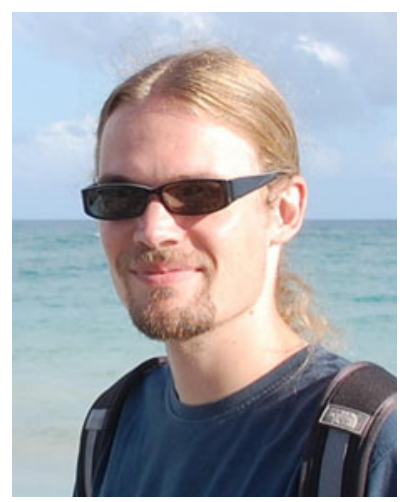

Peter de Bakker graduated in 2007 at the Faculty of Aerospace Engineering of Delft University of Technology. He started as a $\mathrm{PhD}$ candidate at Delft University of Technology in 2008 on the subject of precise point positioning and integrity monitoring. 


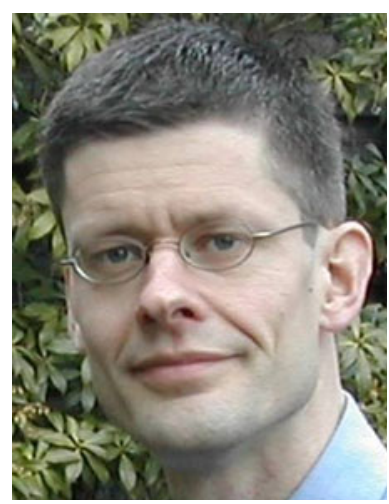

Christian Tiberius is an assistant professor at Delft University of Technology. $\mathrm{He}$ is involved in GNSS positioning and navigation research, with emphasis on data quality control, SBAS, and precise point positioning.

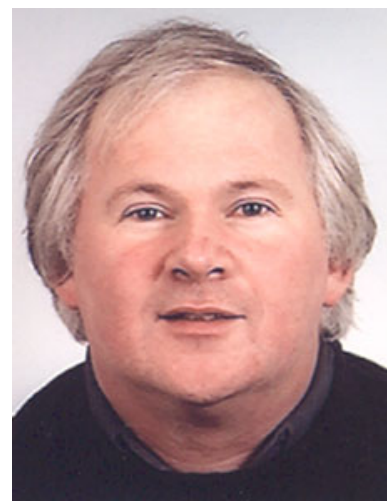

Hans van der Marel is an assistant professor at Delft University of Technology, The Netherlands. He is involved in research on high precision GNSS positioning and navigation using GPS and Galileo, and scientific and meteorological applications of GNSS.

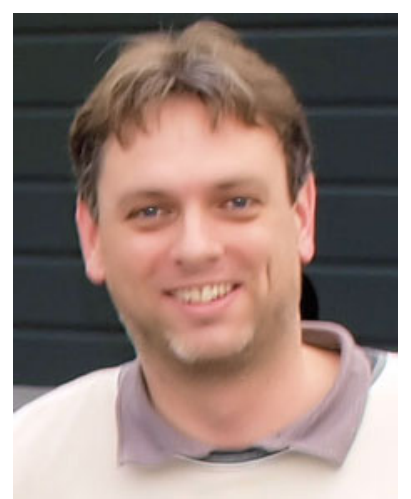

Roel van Bree graduated in Astronomy from Leiden University in the Netherlands in 1997. He has worked for ten years at TNO Defense and Security on Synthetic Aperture Radar. Currently, he is working as a research associate at Delft University of Technology on precise point positioning and navigation with GPS and Galileo. 